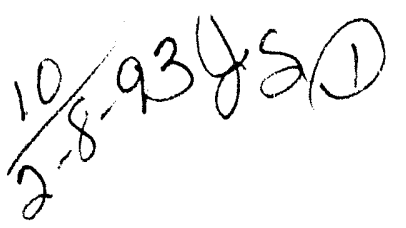

\title{
A Variable Current Bypass Shunt for Electromagnets
}

\author{
M.M. Berndt and J.J. Lipari \\ Stanford Linear Accelerator Center, Stanford, CA*
}

STAC-PUB- -5987

\section{DE93 004273}

\section{Abstract}

An IGBT is used to control the current in bypass circuits that vary the relative strength of magnets connected in series. Each circuit consists of a water cooled assembly rated for continuous operation at 70 Volts and 0-50 Amperes DC, with $1 \mathrm{KV}$ irisulation between control/monitoring elements and magnets. The circuit operates as a $10 \mathrm{KHz}$ PWM switcher that includes filters and transient protection.

\section{INTRODUCTION}

When a number of magnets are connected in series, and it becomes necessary to make small changes in the relative strengths of the magnets, current bypass shunts rather than other schemes, such as trim windings or separate power supplies, are sometimes preferrei. The reasons for this choice may be economic (lower costs) or technical (possibly better tracking during transients).

A bypass shunt that consists only of a resistor, fixed or variable, is seldom satisfactory because the bypass current will not accurately track the magnet current for changes in temperature or in the operating point. For this reason an electronic bypass, using a power transistor as the controlling element, is normally used. Typically the transistor operates in the linear mode, with the resulting power dissipation occurring partly in the transistor and partly in a current limiting resistor in series with the transistor. The power dissipated in the transistor can be quite large in the linear mode, with a maximum value of $P_{\max }=E^{2} / 4 R$, where $E$ is the open circuit voltage

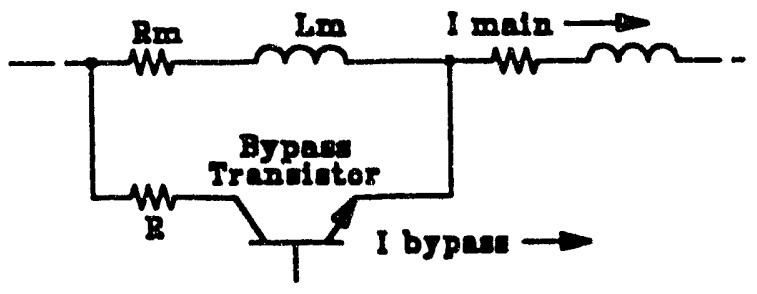

Fig. 1 Transistor controlled magnet bypass circuit. $R$ is a current limiting resistor, and includes cabling.

Work supported by Department of Energy Contract DE-AC0376SF00515

Presented at the Nuclear Science Symposium Orlando, Florida, October 26-31, 1992 across the transistor and $R$ is the total circuit resistance (Fig. 1). If the transistor operates in the switching mode, then the the power it must dissipate is much less for the same average current. Of course filter capacitors will be needed because the bypass current must be continuous, not chopped.

Two prominent problems in the design of a transistor controlled bypass circuit, whether linear or switch-mode, are: (a) Adequate protection of the transistor for routine transients (voltage surge at turnon, or sudden loss of drive to the pass transistor), and (b) an economic yet adequate means of controlling and monitoring the bypass current in the presence of common mode voltages that can be quite large.

\section{DESIGN AND ANALYSIS}

The Main Arc magnets of the SLAC Linear Collider (SLC) are energized by two DC power supplies that routinely run at 4500 Amperes and 280 Volts, although normal operating values are slightly lower. At two places in each of the arcs (North and South) it was necessary to install $1 \%$ bypass circuits around one quarter of the magnets, each bypass rated 70 Volts and 50 Amperes $(3.5 \mathrm{KW})$. Grounding of the magnet circuit is such that control and monitoring of the bypass current occurs in the presence of a maximum DC common mode voltage of 280 Volts.

We decided to use an insulated gate bipolar transistor (IGBT), operating as a $10 \mathrm{KHz}$ pulse width modulated switch (PWM) for controlling each of the four bypass circuits. IGBTs offer low on-state losses, plus simple FET-like gate requirements.

For a valid analysis it is necessary to have an adequate representation of the SLC arc magnets. A Thevenin equivalent circuit for the bypassed magnets consists of a DC voltage source V1 (part of Fig. 2) given by the voltage measured across one-fourth of the magnets when the bypass current is zero, in series with a source impedance $\mathrm{Rm}$ plus $\mathrm{Lm}$. Rm consists of one-fourth of the total arc magnet resistance plus a half mile of cabling from the magnets to the bypass. $\mathrm{Lm}$ is found from the magnet $\mathrm{L} / \mathrm{R}$ time constant (1.5 sec), as measured from the current decay following a power supply shutdown, and is again equal to onefourth the total magnet inductance. The value of $\mathrm{Lm}$ is important for the calculation of transients, because the energy stored in $\mathrm{Lm}$ and the resulting voltage 
spikes are potentially destructive to circuit components, even with only $1 \%$ of the current bypassed.

The basic equivalent circuit used for analysis is shown in Fig 2. V1, Rm and $\mathrm{Lm}$ are the Thevenin equivalent for the magnets plus cabling. $\mathrm{R} 1, \mathrm{Cl}$ and C2 are the filter. The IGBT can be modeled in its simplest form, as an ideal switch S1. R4 is the current limiting resistor with a calculated inductance LA that incorporates cabling inductance. $R 3$ and $C 3$ make up a transient snubber network for S1, and D1 is a fast recovery free wheeling diode.

The $\mathrm{R} 1 / \mathrm{C} 1 / \mathrm{C} 2$ filter combination was chosen after considering such factors as acceptable switcher ripple voltage across the magnets, voltages during a transient, and availability of capacitors of appropriate voltage and current rating. $C I$ is a high frequency $100 \mathrm{uF} / 600 \mathrm{VDC}$ capacitor that carries $97 \%$ of the AC current, up to 50 Amperes, during normal switching at $10 \mathrm{KHz} . \mathrm{C} 2$ is a $2200 \mathrm{uF} / 450 \mathrm{VDC}$ electrolytic capacitor that, together with $R 1$, absorbs the stored energy in Lm whenever the action of S1 is suddenly interrupted for any reason. $\mathrm{Lm}, \mathrm{Rm}, \mathrm{R} 1$ and $\mathrm{C} 2$ make a damped filter at about $20 \mathrm{~Hz}$.

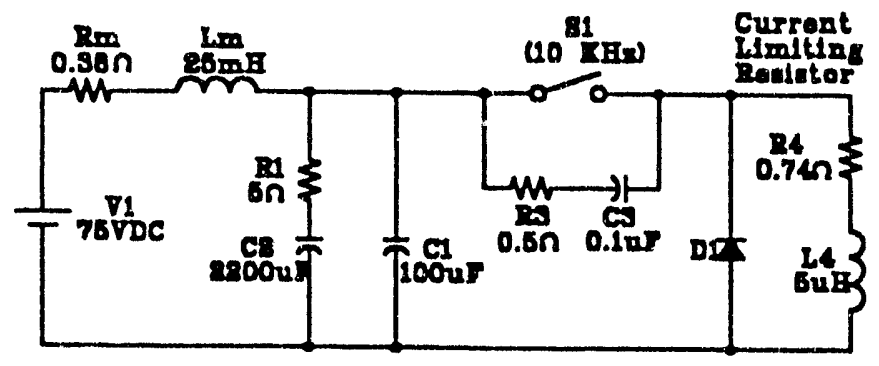

Fig. 2 Equivalent circuit of SLC Arc Magnets with Bypiss.

$$
\begin{aligned}
& \mathrm{Vl}, \mathrm{Rm}, \mathrm{Lm} \text { - Equivalent circuit of bypassed magnet. } \\
& \text { R4, LA _ Current limiting resistor, consisting of } \\
& \mathrm{R} 1, \mathrm{Cl} \text { - Low-frequency transient filter. } \\
& \text { S1 _ IGBT switch, PWM at } 10 \mathrm{KHz} \\
& \mathrm{DI} \text { - Freewheeling diode, in close proximity }
\end{aligned}
$$

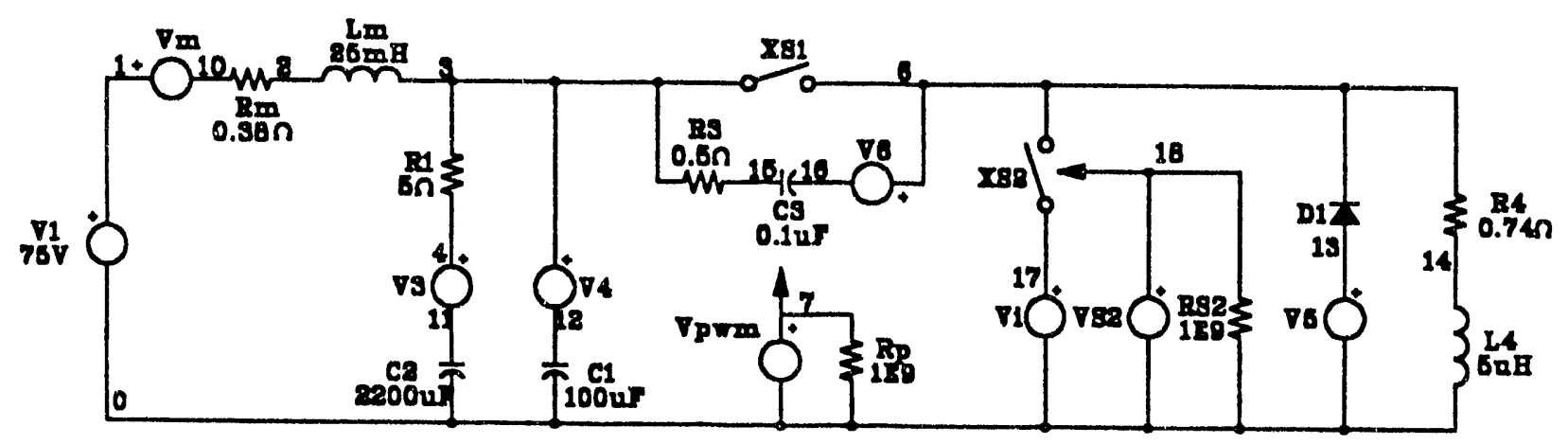

Fig. 3 SPICE model of switcher bypass circuit.

A number of operating modes were investigated using the circuit of Fig 3 for SPICE simulations, in which Vpwm and $R p$ are the switch timing controls. To analyze normal switcher operation using SPICE, it is necessary to know the initial conditions of the circuit at a given duty cycle. But this is difficult when switching times are orders of magnitude faster than filter response. This problem was solved by making an initial estimate of circuit voltages and currents, followed by several iterations, and the addition of the XS2, VS2, RS2 and Vi components. Results are shown in Figures 4 and 5 for operation at $50 \%$ duty cycle, when the resulting ripple across the magnets is maximum.

An analysis of the worst case transient condition is much simpler, since the greatest stress on circuit components occurs when the bypass is at maximum $(100 \%)$, and the drive to $S 1$ is suddenly interrupted. The initial state for this condition is obtained from the
DC solution for S1 closed, without need for the components associated with XS2. The results are shown in Figures 6 and 7. Fig 6 shows that there will be a 340 Volt transient across the switch, considerably higher than the 75 Volt maximum during normal switching. Transient voltages of similar amplitude occur across the magnets and filter capacitors. Fig 7 shows the $C 2$ voltage and current for the same transient condition. C2 carries little current during normal switcher operation, but sees a large current pulse when S1 drive is interrupted.

Other operating modes were also investigated, but are not shown.

\section{CONSTRUCTION}

The four switcher assemblies were built as separate units, each with $\mathrm{Cl}, \mathrm{S1}$ and $\mathrm{D} 1$ in a compact package. 


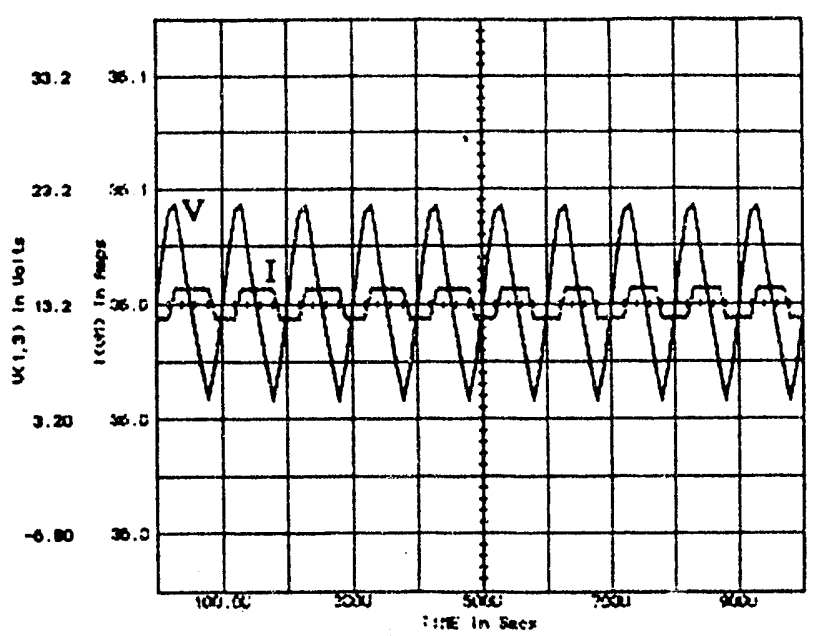

Fig. 4 Ripple in bypass current, and magnet ripple voltage, at $10 \mathrm{KHz}$ and $50 \%$ duty cycle.

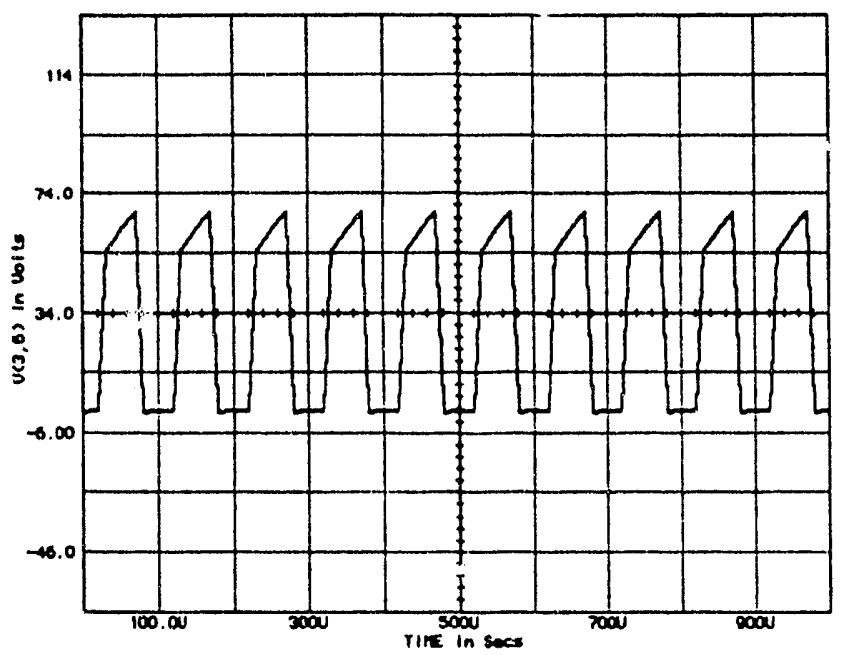

Fig. 5 IGBT voltage at $10 \mathrm{KHz}$ and $50 \%$ duty cycle.

R4 is a water cooled stainless steel resistor, mounted a few feet away. A PWM driver board has all the components that provide the necessary isolation for control and measurement. A Signetics SG3524 switch mode control IC is used to generate the $10 \mathrm{KHz}$ PWM signal, and a hybrid IGBT driver IC, Fuji EXB841, conditions the pulse for the IGBT gate. The EXB841, together with its associated small DC/DC converter, provides isolation for the analog input signal. The bypass current is monitored using a $100 \mathrm{mV}$ current shunt in series with $R \mathrm{~m}$, rather than a Hall effect device, because of better temperature stability. The shunt signal is amplified with two Analog Devices AD210 isolation amplifiers, one for monitoring, the other for feedback. Thus all current control/measurement and error amplification is done at ground potential. The regulator loop is closed for current feedback, using an error signal amplifier and frequency compensation network.

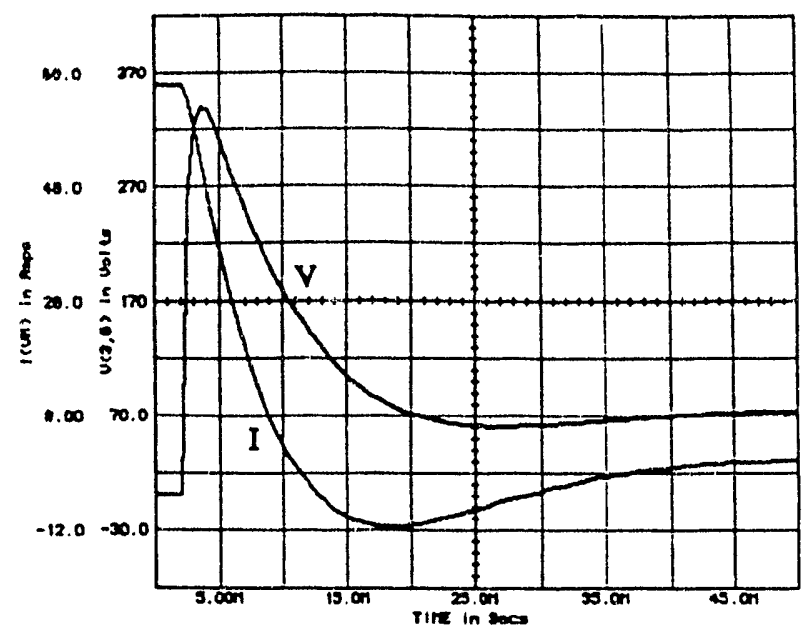

Fig. 6 Bypass current and voltage when drive to $S 1$ is interrupted.

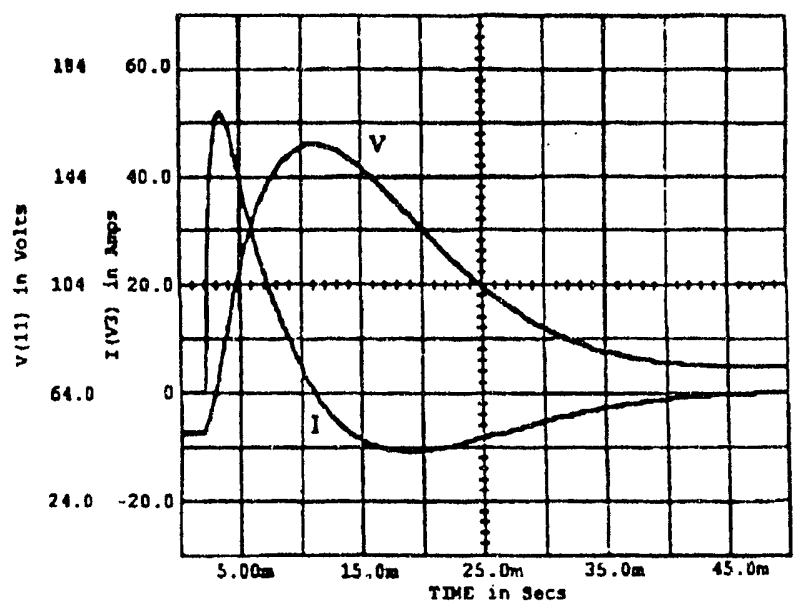

Fig 7 Voltage and current for $\mathrm{C} 2$ when drive to $\mathrm{S} 1$ is interrupted.

\section{OPERATIONAL EXPERIENCE}

Four switcher assemblies were built and installed in the SLC Main Arc magnet system and have performed as predicted. A worst case transient situation was generated by running the bypass at $95 \%$ duty cycle and abruptly suppressing the PWN trigger pulse. The resulting voltage peak across $\mathrm{Cl}$ and $\mathrm{S} 1$ was about 330 Volts, very close to the 340 Volt peak predicted by the SPICE analysis.

\section{REFERENCE}

Mears \& Hymowitz, "Simulating with SPICE", Intusoft Co., San Pedro, CA 

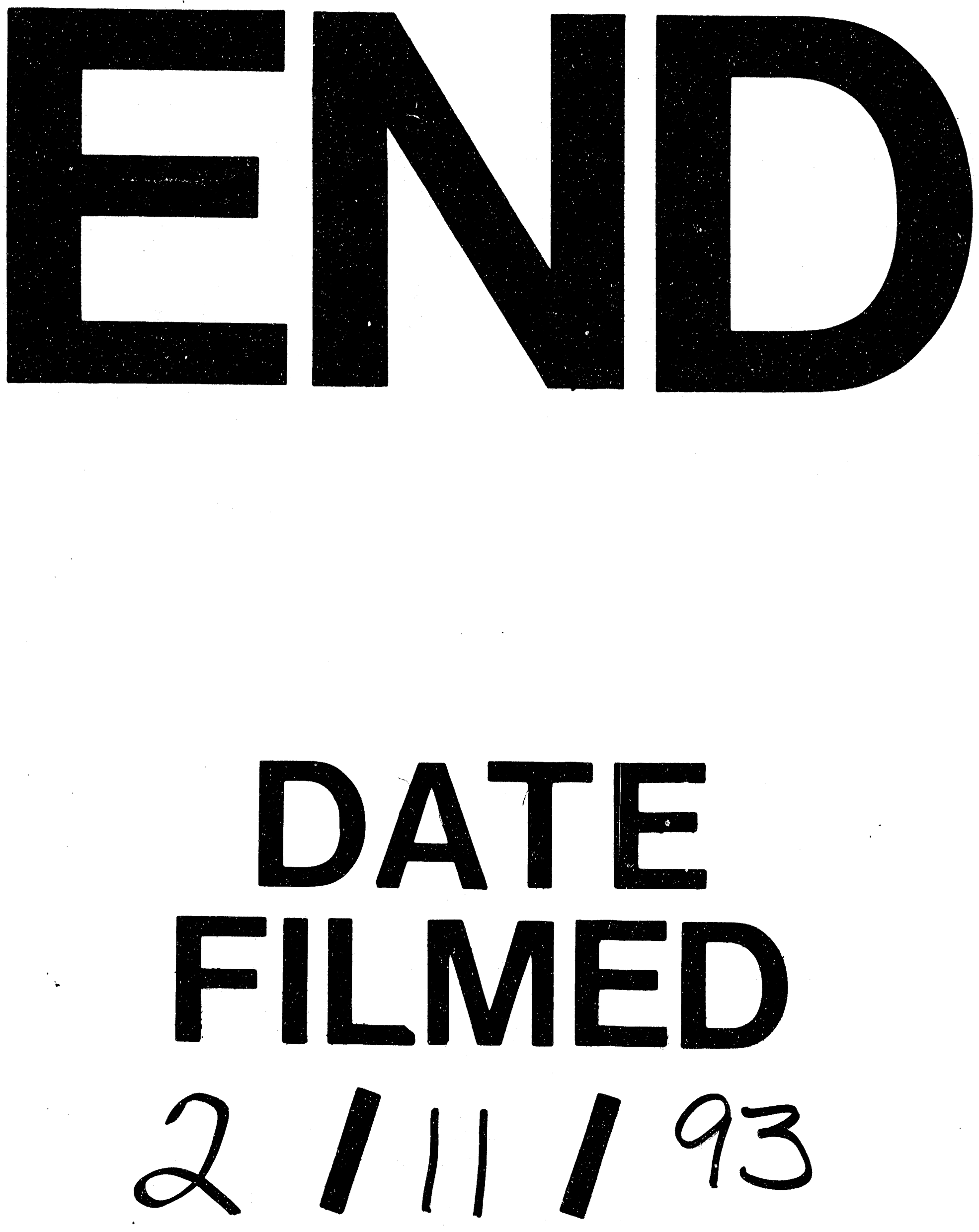
